\title{
Review Article \\ The Effect of Mitochondrial Dysfunction on Cytosolic Nucleotide Metabolism
}

\author{
Claus Desler, ${ }^{1}$ Anne Lykke, ${ }^{2}$ and Lene Juel Rasmussen ${ }^{1}$ \\ ${ }^{1}$ Center for Healthy Aging, Faculty of Health Sciences, University of Copenhagen, 2200 Copenhagen, Denmark \\ ${ }^{2}$ Department of Science, Systems and Models, Roskilde University, 4000 Roskilde, Denmark
}

Correspondence should be addressed to Lene Juel Rasmussen, ljr@mitosci.net

Received 15 June 2010; Accepted 19 July 2010

Academic Editor: Ashis Basu

Copyright (C) 2010 Claus Desler et al. This is an open access article distributed under the Creative Commons Attribution License, which permits unrestricted use, distribution, and reproduction in any medium, provided the original work is properly cited.

\begin{abstract}
Several enzymes of the metabolic pathways responsible for metabolism of cytosolic ribonucleotides and deoxyribonucleotides are located in mitochondria. Studies described in this paper suggest dysfunction of the mitochondria to affect these metabolic pathways and limit the available levels of cytosolic ribonucleotides and deoxyribonucleotides, which in turn can result in aberrant RNA and DNA synthesis. Mitochondrial dysfunction has been linked to genomic instability, and it is possible that the limiting effect of mitochondrial dysfunction on the levels of nucleotides and resulting aberrant RNA and DNA synthesis in part can be responsible for this link. This paper summarizes the parts of the metabolic pathways responsible for nucleotide metabolism that can be affected by mitochondrial dysfunction.
\end{abstract}

\section{Introduction}

Mitochondria are semiautonomous organelles present in almost all eukaryotic cells in quantities ranging from a single copy to several thousand per cell. Important mitochondrial functions include ATP production by oxidative phosphorylation, $\beta$-oxidation of fatty acids, and metabolism of amino acids and lipids. Furthermore, mitochondria have a prominent role in apoptosis. Mitochondrial dysfunction has been linked to genomic instability, and mutations in mitochondrial DNA (mtDNA) have been shown in a wide array of tumors $[1,2]$.

The focus of this paper is on the consequences of mitochondrial function for metabolism of cytosolic ribonucleotides and deoxyribonucleotides. Several of the ratelimiting steps of these metabolic pathways take place in the mitochondria and can be affected by the fitness of the organelle. Disturbance of mitochondrial function therefore has the potential to affect the cytosolic levels of ribonucleotides and deoxyribonucleotides, which in turn can affect the genomic stability.

Where deoxyribonucleotides are exclusively destined for DNA synthesis in the form of deoxyribonucleotides triphosphates (dNTP), ribonucleotides have a multitude of uses in RNA synthesis in the form of ribonucleotide triphosphates (rNTP), as chemical energy transporters in the form of adenine- $5^{\prime}$-triphosphate (ATP) and to form the basis of second messenger molecules. Disruption of the intracellular levels of deoxyribonucleotides or ribonucleotides is unfavorable for the cell. Imbalance of the dNTP pools can induce a variety of genetic changes such as base substitutions, frameshift mutations, delay of replication fork progression and DNA replication, as well as increase the frequency of fragile sites [3-9]. Decreased levels of rNTP pools inhibits RNA synthesis, likely by inhibiting the initiation frequency of RNA polymerase I, and thereby inhibiting the synthesis of rRNA [10]. Furthermore, inhibition of purine and pyrimidine synthesis induces a potentially p53-mediated cell cycle arrest and inhibits proliferation, which ultimately leads to increased cytotoxicity [11-14].

The total number of genes encoding mitochondrial proteins is estimated to 1013 [15]. Mitochondria contain their own autonomous genome, which encodes 13 polypeptides. The remainder is encoded by nuclear DNA and imported into mitochondria. Mitochondrial dysfunction can therefore be the result of mutations in either the mitochondrial or 
nuclear genome. Mitochondria are enclosed by a doublemembrane where the electron transport chain (ETC) maintains an electrochemical potential gradient between the intermembrane space and the matrix of the mitochondria. The ETC is constituted of four membrane bound enzyme complexes (complex I-IV) and two electron carriers (ubiquinon and cytochrome c) and is located in the inner membrane of the mitochondria. The electrochemical gradient is utilized by the ATP synthase (complex V) to generate ATP by oxidative phosphorylation.

The 13 polypeptides encoded by mtDNA constitute critical subunits of the ETC and ATP synthase [16, 17]. Mitochondrial dysfunction resulting from mutations in the mitochondrial genome therefore affects the function of the ETC, the electrochemical gradient and the generation of ATP by oxidative phosphorylation whereas mitochondrial dysfunctions caused by mutations in nuclear encoded proteins can affect all mitochondrial processes.

Several studies have demonstrated a correlation between different types of mitochondrial dysfunction and aberrant synthesis of cytosolic ribonucleotides and deoxyribonucleotides [18-21]. In the following, we will review mitochondrial pathways that are involved in the metabolism of cytosolic ribonucleotides and deoxyribonucleotides. Exempt from this paper is any direct link between aberrant production of ATP from oxidative phosphorylation and synthesis of cytosolic ribonucleotides and deoxyribonucleotides. Most metabolic pathways are dependent on ATP and a review of these processes in response to an insufficient ATP synthesis is outside the scope of this paper.

\section{Dihydroorotate Dehydrogenase Links Mitochondria to the de novo Pyrimidne Biosynthesis}

The flavoenzyme dihydroorotae dehydrogenase (DHODHase) catalyzes the conversion of dihydroorotate to orotate by oxidation, making DHODHase an integral step of the de novo synthesis of pyrimidines. Subsequent catalytic steps convert orotate into uridine monophosphates which can be further converted to UTP and CTP, and ultimately, dTTP and dCTP. DHODHase is located in the inner membrane of the mitochondria with the active site facing the inner membrane [22]. DHODHase is functionally connected to the ETC by a flavin prosthetic group that couples dihydroorotate oxidation to respiratory ubiquinone reduction [23]. From ubiquinol, the reduced form of ubiquinone, the flow of electrons continues through the ETC (see Figure 1).

As a result of the connection between DHODHase and the ETC, it has been suggested that any dysfunction of the ETC-lack of oxygen, presence of inhibitors or mutations of complex III and IV, would entail impairments of the de novo UMP synthesis and a subsequent decrease in the de novo synthesis of pyrimidines and, thereby, the cytosolic ribonucleotide pool [24]. Along this line, it has been suggested that mitochondrial dysfunction will lead to an imbalance of the cytosolic dNTP pool caused by inhibition of the DHODHase [25]. The relationship between DHODHase and the ETC has been described using the two ETC inhibitors, rotenone and sodium cyanide, on mitochondria isolated from rat liver, kidney, and heart. Rotenone inhibits the transfer of electrons from complex I of the ETC to ubiquinone whereas sodium cyanide inhibits complex IV. In isolated mitochondria, treatment with sodium cyanide abolishes the activity of DHODHase while treatment with rotenone does not seem to have an effect [24]. This indicates that only an inhibition or impairment of the complexes of ETC that causes a build up of ubiquinol has an inhibitory effect on the DHODHase. A functional ETC is not only necessary for correct function of DHODHase, but also for correct localization of the protein. DHODHase is encoded by nDNA and the import into the membrane of the mitochondria is dependent on a targeting signal in the N-terminal segment of the protein. It has been demonstrated that import of rat DHODHase into yeast mitochondria was abolished by an uncoupling of the mitochondrial membrane indicating the requirement of a membrane potential for correct positioning of DHODHase [26].

The importance of a functional DHODHase for the de novo synthesis of pyrimidines is emphasized by the effect of inhibitors of DHODHase. Leflunomide and brequinar are two examples of DHODHase inhibitors that bind to the quinone-binding site of DHODHase, thereby, blocking interaction between ubiquinone and the flavin prostethic group of DHODHase [27]. Treatment of human lymphocytes with leflunomide or brequinar arrests the cells in G1 phase and inhibits both RNA and DNA synthesis [28-30]. The inhibitory effects are suppressed by addition of uridine, which can be salvaged to UMP whereby the de novo synthesis of pyrimidines is bypassed. Treating the human leukemic cell line CCRF.CEM with leflunomide or brequninar cause a significant reduction in the levels of CTP and UTP, while the levels of purine nucleotides are unaffected after treatment with lefunomide or increased after treatment with brequinar [31].

Chloramphenicol is an antimicrobial agent that inhibits mitochondrial protein synthesis [32]. Treatment of cells with chloramphenicol therefore mimics an mtDNA induced mitochondrial dysfunction and impaired ETC activity. Treatment of chick embryo cells with chloramphenicol inhibits activity of DHODHase as well as cell growth [33]. Growth of Ehrlich Ascites tumor cells under hypoxic conditions inhibited the ETC and caused reduced activity of the DHODHase and resulted in a G1 arrest [34]. For both the chloramphenicol treated chick embryo cells and hypoxic Ehrlich Ascites tumor cells, growth inhibition was reversed by addition of pyrimidines to the growth media. This indicates that mitochondrial dysfunction affecting the ETC has an inhibitory effect on DHODHase activity that is comparable to inhibition with leflunomide or brequinar. This conclusion is substantiated by the fact that cultured mammalian cells devoid of mtDNA are auxotrophic for pyrimidines and must be routinely grown in the presence of a uridine supplement [21].

An inhibition of DHODHase has been demonstrated to result in decreased levels of pyrimidne ribonucleotides and has been proposed to result in imbalanced dNTP 
pools $[24,25]$. The activity of DHODHase is coupled to the activity of ETC and mitochondrial dysfunctions affecting the activity of the ETC is therefore likely to affect the activity of the DHODase and the de novo synthesis of pyrimidine ribo- and deoxyribonucleotides. In concordance, we have previously demonstrated the human cancer cells depleted of mtDNA had lowered dNTP levels when compared with parental cell lines with functional mtDNA [18]. Furthermore, unpublished data indicates that a leflunomidemediated inhibition of DHODHase in a human cervical cancer cell line results in decreased levels of dTTP and dCTP (data not shown). It is therefore possible that damage to the ETC can affect the cytosolic levels of pyrimidine nucleotides through the activity of the DHODHase.

\section{Mitochondrial Production of One-Carbon Units Links Mitochondria to the de novo Purine Biosynthesis}

Serine is a major source of one-carbon units required for the synthesis of glycine, thymidylate, methionine, several methylation reactions, and, most important for this paper, purine synthesis (see Figure 1). In mammals, serine is derived from the diet and is synthesized from glycolysis via 3phosphoglycerate. Serine is reversibly converted into glycine in a process catalyzed by serine hydroxymethyltransferase (SHMT) [35]. There are two isoenzymes of SHMT, a cytosolic (cSHMT) and a mitochondrial (mSHMT) [36]. It is generally believed that the process catalyzed by mSHMT is the primary pathway for conversion of serine to glycine. This is substantiated by studies using Chinese Hamster Ovary (CHO) cells where loss of mSHMT activity was demonstrated to result in an accumulation of intracellular serine leading to a 15 -fold higher serine concentration. Nevertheless, the net flux through cSHMT was still in the direction of serine synthesis [37]. Furthermore, CHO cells deficient in mSHMT activity are glycine auxotrophs [38], and the auxotrophy can be suppressed by transfection with a human version of $\mathrm{mSHMT}$, suggesting that the primary role of mSHMT but not cSHMT is to generate glycine [39].

During the mSHMT catalyzed conversion of serine to glycine, a methyl group is transferred from serine to tetrahydrofolate (THF), yielding glycine and 5,10-methyleneTHF [35]. Within the mitochondrial compartment, 5,10methyleneTHF is converted into formate in a series of enzymatic conversions initiated by the conversion of 5,10methylene-THF into 5-methyl-THF and subsequently into 10-formyl-THF, in $\mathrm{NAD}^{+}$-dependent reactions catalyzed by the bifunctional enzyme methylene-THF-dehydrogenase. 10 -formyl-THF is converted into formate in a reaction catalyzed by 10 -formyl-THF-synthase and formate can be exported from mitochondria to the cytosol, where it is converted back to 10-formyl-THF in an ATP dependent reaction catalyzed by 10 -formyl-THF-synthetase. Cytosolic 10 -formyl-THF is an essential one-carbon unit donor for the de novo synthesis of purine nucleotides, requiring 2 moles of 10-formyl-THF per mole of purine ring formed Reviewed in $[40,41]$. The role of mitochondrial formate is demonstrated using radioactive labeled variants of formate and serine in murine fibroblasts and human breast cancer cells. Labeled one-carbon units could be traced into both RNA and DNA and their origin could be traced to originate from serine imported to the mitochondria and exported as formate $[19,42]$.

The contribution of mitochondrial formate to purine synthesis is not existing in all cell types. The rate limiting enzyme methylene-THF-dehydrogenase is expressed in transformed and embryonic cell lines as well as undifferentiated cells from bone marrow, thymus, and spleen but not in differentiated tissue from brain, heart, skeletal muscle, liver, or kidney where the mitochondrial production of formate therefore is absent [43]. Even though a supply of mitochondrial produced formate is not necessarily an indispensible carbon source for purine synthesis, a blocked production of formate is demonstrated to inhibit cell-cycle progression in mouse fibroblasts and in phytohemagglutin stimulated splenocytes accompagnied by significant decreases in both cellular deoxyribonucleotide and ribonucleotide pools [19, 20]. Furthermore, mouse embryos that do not express methylene-THF-dehydrogenase and therefore are unable to generate mitochondrial formate are not viable [44], underlining the importance of the mitochohondrial production for formate on nucleotide synthesis in exposed cells.

The proteins necessary for mitochondrial production of one-carbon units and export of formate are all nuclear encoded. This eliminates the possibility of any direct involvement of mitochondrial encoded proteins in this pathway. It is, however, possible that mutations of the mitochondrial genome that will result in a decreased function of the ETC, turnover of intermediates or the production of ATP can have an indirect effect on the mitochondrial production of one-carbon units and formate export. Loss of mtDNA and resulting damaged ETC has been argumented to cause a decrease in mitochondrial NADH oxidation to $\mathrm{NAD}^{+}$and a resulting inhibition on mitochondrial processes dependent on $\mathrm{NAD}^{+}[45]$. Since methylene-THF-dehydrogenase is a $\mathrm{NAD}^{+}$-dependent enzyme, lack of $\mathrm{NAD}^{+}$could limit the production of mitochondrial formate, and it is therefore possible that loss of mtDNA indirectly can influence the production of mitochondrial formate and thereby cytosolic purine synthesis. By comparing human osteosarcoma cells devoid of mtDNA with their parental mtDNA containing cells, it was found that both cell lines grew equally well in complete and glycine-deficient media, demonstrating that the cells without mtDNA were not glycine auxotrophs [19]. This indicates that loss of mtDNA does not abolish purine synthesis, however, it does not remove the possibility that loss of mtDNA limits the production of mitochondrial formate and results in lowered levels of cytosolic purine levels.

\section{Mitochondrial Production of Nitric Oxide Indirectly Affects the Nucleotide Metabolism}

Nitric oxide (NO) is an uncharged and highly diffusible inorganic signal molecule with a wide variety of roles in the organism. Mitochondrial nitric oxide synthase (mtNOS) 


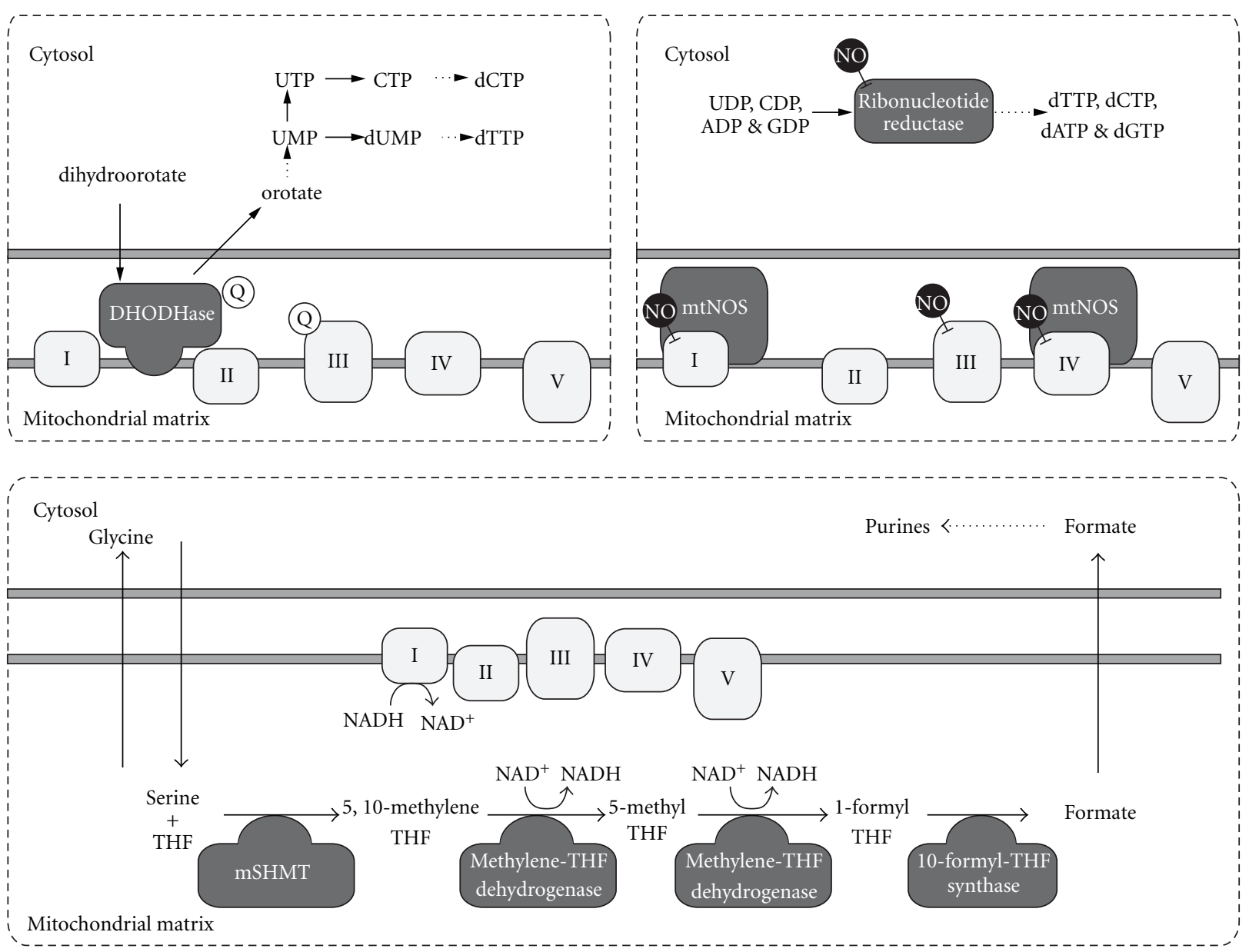

FIGURE 1: Overview of metabolic pathways responsible for nucleotide metabolism that can be affected by mitochondrial dysfunction. The illustration depicts the outer and inner mitochondrial membrane. Complexes of the electron transport chain are illustrated with boxes marked with numerals I-IV. Boxes marked with V depict ATP synthase. Darkened boxes illustrate enzymes of interest. Upper left: Dihydroorotate (DHODHase) is an integral enzyme in the de novo synthesis of pyrimidines. The enzyme is located in the inner mitochondrial membrane and its activity is dependent on an active electron transport chain. Upper right: Mitochondrial nitric oxide synthase (mtNOS) creates NO in a $\mathrm{Ca}^{2+}$ dependent reaction. The enzyme is located in the inner mitochondrial membrane where it interacts with both complexes I and IV of the electron transport chain. The activity of mtNOS is linked to the mitochondrial membrane potential. NO has the potential to inhibit the activity of Complexes I, III, and IV, but also ribonucleotide reductase in the cytosol. Lower: Illustration of the mitochondrial production of one-carbon units and mitochondrial export of formate. See text for details.

is a constitutively expressed nuclear encoded enzyme that generates $\mathrm{NO}$ in a $\mathrm{Ca}^{2+}$-dependent reaction. Furthermore, judging from proven properties of other nitric oxide synthases, production of NO by mtNOS is likely regulated by acetylation and phosphorylation of the enzyme [reviewed in 46]. MtNOS is located in the inner membrane of the mitochondria, [46] where it physically interacts with both complexes I and IV of the ETC (See Figure 1) [47, 48]. NO has at sub-micromolar concentrations been demonstrated to act as a competitive inhibitor of complex IV and to inhibit electron transport at complex III. Furthermore, complex I is inhibited after long-term exposure to NO [49-51] As a result of the inhibitory effects of NO on different sites of the ETC, both the activities of DHODHase and ATP synthase have been demonstrated to be inhibited in response to increased NO levels $[52,53]$. Both the localization of mtNOS and the inhibitory effects of NO on the generation of ATP by oxidative phosphorylation have lead to the general belief that the purpose of mtNOS is to regulate the oxygen uptake according to the energy requirement of the cell in a shortterm fashion. Besides a direct regulatory effect on oxygen uptake, NO has in turn the potential of indirectly inhibiting the synthesis of nucleotides through its inhibitory effects on the DHODHase as discussed previously.

Efflux of NO from the mitochondria is maximal after treatment with oligomycin and minimal after treatment of CCCP. Treatment with oligomycin inhibits the ATP synthase and produces a hyper polarization of the mitochondrial membrane. Conversely, treatment with CCCP uncouples the membrane. This led to the hypothesis that mtNOS is a voltage-dependent enzyme, whose activity is regulated by mitochondrial membrane potential $[54,55]$. Very little is known about any possible deregulation of mtNOS resulting from mitochondrial dysfunction. If the activity of mtNOS 
is linked to the membrane potential and a depolarization inactivates the enzyme, damage to the ETC will therefore inactivate the protein. Conversely inhibition of the ATP synthase leading to a hyperpolarization will activate mtNOS. Examples of mutations affecting the ATP synthase are known. The ATP synthase has been demonstrated to be severely impaired in patients with mutated versions of the mtDNA encoded ATPase 6 subunit of the ATP synthase [56]. The influence of this and other damages to the ATP synthase on mtNOS has, however, not been investigated.

Nitric oxide produced by mtNOS is most likely not constricted to the mitochondria, but may serve a role in the cytosolic compartment. Efflux of $\mathrm{NO}$ from the mitochondria to the cytosol has been demonstrated. This efflux is correlated with the membrane potential of the mitochondria and has been hypothesized to function as a mitochondrial signal to the cytosol, reporting on the energy status of the mitochondria $[54,55]$. By using mitochondria extracted from different rat tissue, the fraction of cytosolic NO resulting from a mitochondrial efflux was calculated to range from $61 \%$ in heart tissue to $18 \%$ in brain tissue [54]. The role of an efflux of NO from the mitochondria into the cytosol is unknown however, it has been suggested that it may serve a regulatory role in cell metabolism and proliferation $[54,57]$. In this case, an imbalance of the dNTP pool demonstrated in cells with a mitochondrial dysfunction could be caused by a resulting error in this pleiotropic regulation of cell metabolism. However, NO have several targets in the cellular compartment, and depending on the amount of NO emanating from the mitochondria, these could provide a more direct relationship between the mitochondria and dNTP balance. During the de novo pathway the reduction of ribonucleotides to $2^{\prime}$-deoxyribonucleotides is catalyzed by the rate limiting enzyme ribonucleotide reductase. The reductase constitutes the major regulator of the de novo dNTP synthesis [58] mediating a reduction of the $2^{\prime}$-hydroxy group in the ribose of the ribonucleotides [59]. The resulting deoxyribonucleotides dADP, dCDP, and dGDP undergo phosphorylation yielding the corresponding dNTPs; however, dTTP is synthesized from dCDP, and dUDP and requires additional steps. Ribonucleotide reductase has been demonstrated to be directly inhibited by NO resulting in a depletion of dNTP proportional to the amount of NO. Treatment of a human lymphoblastoid cell line with different NO prodrugs induced a dNTP imbalance that was comparable to the dNTP profile after treatment with the ribonucleotide reductase inhbitor hydroxy urea [60]. Inhibition of the reductase by $\mathrm{NO}$ was demonstrated to induce a decrease of dATP and dCTP levels whereas the dTTP levels were transiently increased [60].

\section{Flux of Deoxyribonucleotides between Mitochondria and the Cytosol Is Unlikely to Cause a Cytosolic dNTP Imbalance}

The cellular content of rNTP and dNTP is sequestered into two pools, a mitochondrial and a cytosolic, separated by the mitochondrial double membrane [61]. The existence of a mitochondrial salvage pathway of dNTP is well-established whereas the existence of a mitochondrial de novo pathway of dNTP is debated [62]. The mitochondrial inner membrane is impermeable to charged molecules, and consequently, there is no direct exchange of rNTP or dNTP between the cytosolic and mitochondrial compartments. However, several studies have shown a facilitated transport of dNTP between the two compartments reviewed in [62]. Furthermore, export of dNTP from mitochondria and incorporation into nDNA has been demonstrated [63]. It can, therefore, be speculated that the mitochondrial production of dNTP complements the cytosolic dNTP pools and dysfunction of mitochondrial $\mathrm{dNTP}$ synthesis results in a cytosolic dNTP imbalance.

Both de novo and salvage pathways carry out synthesis of cytosolic dNTP, while only the salvage pathway has been demonstrated in mitochondria. The replication of mtDNA is not synchronized with the synthesis of $\mathrm{nDNA}$ [64] and therefore this organelle requires a constant supply of dNTP. Mitochondria are able to synthesize dNTP by a specific salvage pathway where imported deoxyribonucleosides are phosphorylated by the two rate-determining deoxyribonucleoside kinases: thymidine kinase 2 (TK2) and deoxyguanosine kinase (dGK) yielding deoxyribonucleoside mono-phosphates, which in turn can be further phosphorylated to their corresponding di- and triphosphates or be dephosphorylated to deoxyribonucleosides again by mitochondrial deoxyribonucleotidases $[65,66]$. The presence of a mitochondrial located ribonucleotide reductase has been proposed $[62,66]$, opening for the possibility of a mitochondrial de novo synthesis of dNTP. The contribution of this pathway to the mitochondrial and cytosolic pool of dNTP is however unknown.

Export of deoxyribonucleotides from the mitochondrial to the cytosolic compartment has been demonstrated. In cells lacking essential enzymes of the cytosolic salvage pathway, an exogenous source of labeled thymidine was imported into the mitochondria and phosporylated exclusively by the mitochondrial salvage pathway, resulting in a mitochondrial pool of labeled dTTP. Efflux to the cytosolic compartment and incorporation of labeled dTTP into nDNA could be demonstrated, confirming an export of mitochondrial deoxyribonucleotides. Even though the cells lacked a functional cytosolic salvage pathway the contribution of the labeled dTTP only constituted a fraction of the cytosolic pool of dNTP, indicating a strong dominance of dNTP produced by the de novo pathway, demonstrating that the mitochondrial contribution to the cytosolic pool of dNTP is negligible [63].

In nondividing tissue, loss of function mutations of TK2 has been demonstrated to result in mitochondrial dNTP imbalance and consequently deletions and depletions of mtDNA [67]. Furthermore, even minor impairment of TK2 activity has been reported to result in a decreased mitochondrial dNTP pool [68]. Using a human cervical cancer cell line that can be induced to express lower levels of TK2, it was possible to reduce the activity of TK2 in the cell with $47 \%$ [69]. The activity was assayed by the ability of TK2 to phosphorylate thymidine- $\beta$-D-arabinoside. Decreasing the activity of the mitochondrial salvage pathway 
by lowering the level of TK2 did not alter the cytosolic dNTP pools, supporting the small contribution of mitochondrial dNTP export to the cellular dNTP pool [69].

Whereas the mitochondrial export of dNTP, most likely is an insignificant contribution to the cytosolic dNTP pools, import of dNTP to the mitochondria is essential for the fidelity of mtDNA. In cycling cells, the majority of the intramitochondrial deoxynucleotides are synthesized in the cytosol and imported into mitochondria [63]. The p53 inducible small subunit of the ribonucleotide reductase p53R2 is believed to allow de novo synthesis of dNTP outside the S-phase in response to DNA damage [70]. Mutations of p53R2 have been demonstrated to result in mtDNA depletion, likely caused by insufficient mitochondrial dNTP pools [71]. This indicates that mitochondria is dependent of cytosolic dNTP in all phases of cell cycle.

In summary, published data from the literature show that export of mitochondrial-produced dNTP is negligible compared to the cytosolic dNTP synthesis and the flux of dNTP is most likely in the direction of from the cytosol to the mitochondria. Furthermore, inhibition of the mitochondrial salvage pathway has no effect on the cytosolic dNTP pools. Together, this strongly suggests that dysfunction of mitochondrial salvage, or potentially de novo, synthesis of dNTP is unlikely to cause a cytosolic dNTP imbalance by deficient export or excessive import of deoxyribonucleotides.

\section{Conclusion}

In this paper we describe three mitochondrial pathways involved in the synthesis of cytosolic rNTP and dNTP. These three pathways encompass (1) DHODHase-mediated conversion of dihydroorotate to orotate, a rate limiting step of de novo synthesis of pyrimidines; (2) export of mitochondrial one carbon units in the form of formate, used in the production of cytosolic purine nucleotides; and (3) mitochondrial produced NO acting as an effector with both intra mitochondrial and cytosolic targets involved in the cytosolic synthesis of rNTP and dNTP.

The mitochondrial production and export of formate diverge from the pathways involving DHODHase and mitochondrial NO. All components responsible for mitochondrial production and export of formate are nuclear encoded and the pathway is sensitive to mutations in the nuclear genes encoding these. However, even though the pathway can be limited by comprehensive damage to the ETC, the pathway is most likely substrate-dependent and not regulated by the overall activity of the mitochondria. In contrast, the activity of DHODHase is directly dependent on the activity of the ETC and the membrane potential. In this regard, the activity of DHODHase reflects the activity of the mitochondria. Even though DHODHase is nuclear encoded, the ETC consists of both mitochondrial and nuclear encoded subunits. A degradation of mtDNA as seen in a multitude of pathological conditions including cancer [1,2], or as demonstrated in aged tissue [72] will therefore affect the activity of DHODHase and consequently the synthesis of pyrimidine nucleotides. In contrast, mitochondrial export of formate and related purine synthesis will likely be unaffected by nothing less than a complete degradation of mtDNA and the ETC. NO produced from mtNOS targets both the DHODHase and ATPase and is proposed to be a metabolic feedback mechanism regulated by the mitochondrial membrane potential. By inhibiting the DHODHase, mtNOS indirectly affects the de novo synthesis of pyrimidines. MtNOS also releases NO into the cytosolic compartment and can potentially target the ribonucleotide reductase and thereby inhibit the major regulator of the de novo synthesis of dNTP. It was demonstrated that mtNOS is activated by hyper polarization of the mitochondrial membrane. A mitochondrial dysfunction, like damage to the ATP synthase, that allows an excessive build up of mitochondrial membrane potential is therefore likely to activate mtNOS whereas a general degradation of mtDNA and/or the ETC leads to a depolarization of the mitochondrial memebrane and inactivate the enzyme.

The effect of especially DHODHase, but also mtNOS and to some extent mitochondrial production of formate, is dependent on the activity of ETC and mitochondrial membrane potential. In functional cells, this pathway probably constitutes at feedback mechanism, where synthesis of RNA and DNA is regulated by the mitochondrial activity. However, in a pathological context, this relationship has the potential of affecting the genomic stability of the nuclear genome. Mutations affecting the activity of the ETC can lead to a detrimental effect on the cytosolic dNTP levels, and in turn imbalanced dNTP pools can cause genetic instability in the nuclear genome. In accordance, we have demonstrated that human cell lines devoid of mtDNA showed decreased and imbalanced levels of dNTP as well as increased frequency of chromosomal instability compared to parental cell lines with functional mtDNA $[18,73]$. Chromosomal instability has been demonstrated to occur in human osteosarcoma cell lines devoid of mtDNA. Furthermore, the cells were demonstrated to have tumorigenic properties measured by an increased anchorage independent growth when compared to parental cells with functional mtDNA. It was reported that the reintroduction of functional mitochondria with mtDNA could suppress the tumorigenic phenotype [74]. These observations and others define a role for dysfunctional mitochondria in the progression of cancer. It will be important to further elucidate the role of the mitochondrial function on the dNTP regulation in the detrimental relationship between dysfunctional mitochondria and cancer due to the known mutagenic effects of dNTP imbalance.

\section{References}

[1] J. S. Penta, F. M. Johnson, J. T. Wachsman, and W. C. Copeland, "Mitochondrial DNA in human malignancy," Mutation Research, vol. 488, no. 2, pp. 119-133, 2001.

[2] J. S. Modica-Napolitano and K. K. Singh, "Mitochondria as targets for detection and treatment of cancer," Expert Reviews in Molecular Medicine, vol. 4, no. 9, pp. 1-19, 2002. 
[3] P.-Y. Ke, Y.-Y. Kuo, C.-M. Hu, and Z.-F. Chang, "Control of dTTP pool size by anaphase promoting complex/cyclosome is essential for the maintenance of genetic stability," Genes and Development, vol. 19, no. 16, pp. 1920-1933, 2005.

[4] P. Reichard, "Interactions between deoxyribonucleotide and DNA synthesis," Annual Review of Biochemistry, vol. 57, pp. 349-374, 1988.

[5] K. Bebenek and T. A. Kunkel, "Frameshift errors initiated by nucleoside misincorporation," Proceedings of the National Academy of Sciences of the United States of America, vol. 87, no. 13, pp. 4946-4950, 1990.

[6] P. B. Jacky, B. Beek, and G. R. Sutherland, "Fragile sites in chromosomes: possible model for the study of spontaneous chromosome breakage," Science, vol. 220, no. 4592, pp. 69-70, 1983.

[7] B. A. Kunz and S. E. Kohalmi, "Modulation of mutagenesis by deoxyribonucleotide levels," Annual Review of Genetics, vol. 25, pp. 339-359, 1991.

[8] R. G. Wickremasinghe and A. V. Hoffbrand, "Reduced rate of DNA replication fork movement in megaloblastic anemia," Journal of Clinical Investigation, vol. 65, no. 1, pp. 26-36, 1980.

[9] B. Golos and J. Malec, "Comparison of the effect of hydroxyurea and methotrexate on DNA fragmentation at various reaction conditions," Neoplasma, vol. 38, no. 6, pp. 559-564, 1991.

[10] I. Grummt and F. Grummt, "Control of nucleolar RNA synthesis by the intracellular pool sizes of ATP and GTP," Cell, vol. 7, no. 3, pp. 447-453, 1976.

[11] M. Kondo, T. Yamaoka, S. Honda et al., "The rate of cell growth is regulated by purine biosynthesis via ATP production and G1 to S phase transition," Journal of Biochemistry, vol. 128, no. 1, pp. 57-64, 2000.

[12] L. Quéméneur, L.-M. Gerland, M. Flacher, M. Ffrench, J.P. Revillard, and L. Genestier, "Differential control of cell cycle, proliferation, and survival of primary T lymphocytes by purine and pyrimidine nucleotides," Journal of Immunology, vol. 170, no. 10, pp. 4986-4995, 2003.

[13] S. P. Linke, K. C. Clarkin, A. Di Leonardo, A. Tsou, and G. M. Wahl, "A reversible, p53-dependent G0/G1 cell cycle arrest induced by ribonucleotide depletion in the absence of detectable DNA damage," Genes and Development, vol. 10, no. 8, pp. 934-947, 1996.

[14] L. L. Bennett Jr., D. Smithers, and L. M. Rose, "Inhibition of synthesis of pyrimidine nucleotides by 2-hydroxy-3-(3,3dichloroallyl)-1,4-naphthoquinone," Cancer Research, vol. 39, no. 12 , pp. 4868-4874, 1979.

[15] D. J. Pagliarini, S. E. Calvo, B. Chang et al., "A mitochondrial protein compendium elucidates complex I disease biology," Cell, vol. 134, no. 1, pp. 112-123, 2008.

[16] D. C. Wallace, "Mitochondrial diseases in man and mouse," Science, vol. 283, no. 5407, pp. 1482-1488, 1999.

[17] S. DiMauro and E. A. Schon, "Mitochondrial respiratorychain diseases," The New England Journal of Medicine, vol. 348, no. 26, pp. 2656-2668, 2003.

[18] C. Desler, B. Munch-Petersen, T. Stevnsner et al., "Mitochondria as determinant of nucleotide pools and chromosomal stability," Mutation Research, vol. 625, no. 1-2, pp. 112-124, 2007.

[19] H. Patel, E. Di Pietro, and R. E. MacKenzie, "Mammalian fibroblasts lacking mitochondrial $\mathrm{NAD}^{+}$-dependent methylenetetrahydrofolate dehydrogenase-cyclohydrolase are glycine auxotrophs," The Journal of Biological Chemistry, vol. 278, no. 21, pp. 19436-19441, 2003.
[20] S. J. James, B. J. Miller, D. R. Cross, L. J. McGarrity, and S. M. Morris, "The essentiality of folate for the maintenance of deoxynucleotide precursor pools, DNA synthesis, and cell cycle progression in PHA-stimulated lymphocytes," Environmental Health Perspectives, vol. 101, no. 5, pp. 173-178, 1993.

[21] M. P. King and G. Attardi, "Human cells lacking mtDNA: repopulation with exogenous mitochondria by complementation," Science, vol. 246, no. 4929, pp. 500-503, 1989.

[22] M. E. Jones, "The genes for and regulation of the enzyme activities of two multifunctional proteins required for the de novo pathway for UMP biosynthesis in mammals," Molecular Biology, Biochemistry, and Biophysics, vol. 32, pp. 165-182, 1980.

[23] B. Bader, W. Knecht, M. Fries, and M. Löffler, "Expression, purification, and characterization of histidine-tagged rat and human flavoenzyme dihydroorotate dehydrogenase," Protein Expression and Purification, vol. 13, no. 3, pp. 414-422, 1998.

[24] M. Löffler, J. Jöckel, G. Schuster, and C. Becker, "Dihydroorotat-ubiquinone oxidoreductase links mitochondria in the biosynthesis of pyrimidine nucleotides," Molecular and Cellular Biochemistry, vol. 174, no. 1-2, pp. 125-129, 1997.

[25] K. K. Singh, "Mitochondria damage checkpoint in apoptosis and genome stability," FEMS Yeast Research, vol. 5, no. 2, pp. 127-132, 2004.

[26] J. Rawls, W. Knecht, K. Diekert, R. Lill, and M. Löffler, "Requirements for the mitochondrial import and localization of dihydroorotate dehydrogenase," European Journal of Biochemistry, vol. 267, no. 7, pp. 2079-2087, 2000.

[27] S. Liu, E. A. Neidhardt, T. H. Grossman, T. Ocain, and J. Clardy, "Structures of human dihydroorotate dehydrogenase in complex with antiproliferative agents," Structure, vol. 8, no. 1, pp. 25-33, 2000.

[28] A. S.-F. Chong, K. Rezai, H. M. Gebel et al., "Effects of leflunomide and other immunosuppressive agents on $\mathrm{T}$ cell proliferation in vitro," Transplantation, vol. 61, no. 1, pp. 140145, 1996.

[29] K. Rückemann, L. D. Fairbanks, E. A. Carrey et al., "Leflunomide inhibits pyrimidine de novo synthesis in mitogenstimulated T-lymphocytes from healthy humans," The Journal of Biological Chemistry, vol. 273, no. 34, pp. 21682-21691, 1998.

[30] S. Greene, K. Watanabe, J. Braatz-Trulson, and L. Lou, "Inhibition of dihydroorotate dehydrogenase by the immunosuppressive agent leflunomide," Biochemical Pharmacology, vol. 50, no. 6, pp. 861-867, 1995.

[31] H. M. Cherwinski, N. Byars, S. J. Ballaron, G. M. Nakano, J. M. Young, and J. T. Ransom, "Leflunomide interferes with pyrimidine nucleotide biosynthesis," Inflammation Research, vol. 44, no. 8, pp. 317-322, 1995.

[32] B. Storrie and G. Attardi, "Expression of the mitochondrial genome in HeLa cells. IX. Effect of inhibition of mitochondrial protein synthesis on mitochondrial formation," Journal of Cell Biology, vol. 56, no. 3, pp. 819-831, 1973.

[33] M. Grégoire, R. Morais, M. A. Quilliam, and D. Gravel, "On auxotrophy for pyrimidines of respiration-deficient chick embryo cells," European Journal of Biochemistry, vol. 142, no. 1, pp. 49-55, 1984.

[34] M. Löffler, "On the role of dihydroorotate dehydrogenase in growth cessation of Ehrlich ascites tumor cells cultured under oxygen deficiency," European Journal of Biochemistry, vol. 107, no. 1 , pp. 207-215, 1980. 
[35] L. Schirch, "Serine hydroxymethyltransferase," Advances in Enzymology and Related Areas of Molecular Biology, vol. 53, pp. 83-112, 1982.

[36] T. A. Garrow, A. A. Brenner, V. M. Whitehead et al., "Cloning of human cDNAs encoding mitochondrial and cytosolic serine hydroxymethyltransferases and chromosomal localization," The Journal of Biological Chemistry, vol. 268, no. 16, pp. 11910-11916, 1993.

[37] M. R. Narkewicz, S. D. Sauls, S. S. Tjoa, C. Teng, and P. V. Fennessey, "Evidence for intracellular partitioning of serine and glycine metabolism in Chinese hamster ovary cells," Biochemical Journal, vol. 313, no. 3, pp. 991-996, 1996.

[38] W. Pfendner and L. I. Pizer, "The metabolism of serine and glycine in mutant lines of Chinese hamster ovary cells," Archives of Biochemistry and Biophysics, vol. 200, no. 2, pp. 503-512, 1980.

[39] P. J. Stover, L. H. Chen, J. R. Suh, D. M. Stover, K. Keyomarsi, and B. Shane, "Molecular cloning, characterization, and regulation of the human mitochondrial serine hydroxymethyltransferase gene," The Journal of Biological Chemistry, vol. 272, no. 3, pp. 1842-1848, 1997.

[40] D. R. Appling, "Compartmentation of folate-mediated onecarbon metabolism in eukaryotes," FASEB Journal, vol. 5, no. 12, pp. 2645-2651, 1991.

[41] F. Depeint, W. R. Bruce, N. Shangari, R. Mehta, and P. J. O'Brien, "Mitochondrial function and toxicity: role of B vitamins on the one-carbon transfer pathways," ChemicoBiological Interactions, vol. 163, no. 1-2, pp. 113-132, 2006.

[42] T.-F. Fu, J. P. Rife, and V. Schirch, "The role of serine hydroxymethyltransferase isozymes in one-carbon metabolism in MCF-7 cells as determined by 13C NMR," Archives of Biochemistry and Biophysics, vol. 393, no. 1, pp. 42-50, 2001.

[43] N. R. Mejia and R. E. MacKenzie, "NAD-dependent methylenetetrahydrofolate dehydrogenase is expressed by immortal cells," The Journal of Biological Chemistry, vol. 260, no. 27, pp. 14616-14620, 1985.

[44] E. Di Pietro, J. Sirois, M. L. Tremblay, and R. E. MacKenzie, "Mitochondrial NAD-dependent methylenetetrahydrofolate dehydrogenase-methenyltetrahydrofolate cyclohydrolase is essential for embryonic development," Molecular and Cellular Biology, vol. 22, no. 12, pp. 4158-4166, 2002.

[45] R. K. Naviaux, "Mitochondrial control of epigenetics," Cancer Biology and Therapy, vol. 7, no. 8, pp. 1191-1193, 2008.

[46] A. Tatoyan and C. Giulivi, "Purification and characterization of a nitric-oxide synthase from rat liver mitochondria," The Journal of Biological Chemistry, vol. 273, no. 18, pp. 1104411048, 1998.

[47] T. Persichini, V. Mazzone, F. Polticelli et al., "Mitochondrial type I nitric oxide synthase physically interacts with cytochrome c oxidase," Neuroscience Letters, vol. 384, no. 3, pp. 254-259, 2005.

[48] M. C. Franco, V. G. Antico Arciuch, J. G. Peralta et al., "Hypothyroid phenotype is contributed by mitochondrial complex I inactivation due to translocated neuronal nitricoxide synthase," The Journal of Biological Chemistry, vol. 281, no. 8, pp. 4779-4786, 2006.

[49] M. W. J. Cleeter, A. M. Cooper, B. M. Darley-Usmar, D. Moncada, and A. H. V. Schapira, "Reversible inhibition of cytochrome $\mathrm{c}$ oxidase, the terminal enzyme of the mitochondrial respiratory chain, by nitric oxide," FEBS Letters, vol. 345, no. 1, pp. 50-54, 1994.
[50] J. J. Poderoso, M. C. Carreras, C. Lisdero, N. Riobó, F. Schöpfer, and A. Boveris, "Nitric oxide inhibits electron transfer and increases superoxide radical production in rat heart mitochondria and submitochondrial particles," Archives of Biochemistry and Biophysics, vol. 328, no. 1, pp. 85-92, 1996.

[51] N. A. Riobó, E. Clementi, M. Melani et al., "Nitric oxide inhibits mitochondrial NADH:ubiquinone reductase activity through peroxynitrite formation," Biochemical Journal, vol. 359, no. 1, pp. 139-145, 2001.

[52] C. Beuneu, R. Auger, M. Löffler, A. Guissani, G. Lemaire, and M. Lepoivre, "Indirect inhibition of mitochondrial dihydroorotate dehydrogenase activity by nitric oxide," Free Radical Biology and Medicine, vol. 28, no. 8, pp. 1206-1213, 2000.

[53] P. S. Brookes, J. P. Bolaños, and S. J. R. Heales, "The assumption that nitric oxide inhibits mitochondrial ATP synthesis is correct," FEBS Letters, vol. 446, no. 2-3, pp. 261263, 1999.

[54] A. Boveris, L. B. Valdez, T. Zaobornyj, and J. Bustamante, "Mitochondrial metabolic states regulate nitric oxide and hydrogen peroxide diffusion to the cytosol," Biochimica et Biophysica Acta, vol. 1757, no. 5-6, pp. 535-542, 2006.

[55] L. B. Valdez, T. Zaobornyj, and A. Boveris, "Mitochondrial metabolic states and membrane potential modulate mtNOS activity," Biochimica et Biophysica Acta, vol. 1757, no. 3, pp. 166-172, 2006.

[56] A. Baracca, S. Barogi, V. Carelli, G. Lenaz, and G. Solaini, "Catalytic activities of mitochondrial ATP synthase in patients with mitochondrial DNA T8993G mutation in the ATPase 6 gene encoding subunit a," The Journal of Biological Chemistry, vol. 275, no. 6, pp. 4177-4182, 2000.

[57] A. Boveris, L. E. Costa, E. Cadenas, and J. J. Poderoso, "Regulation of mitochondrial respiration by adenosine diphosphate, oxygen, and nitric oxide," Methods in Enzymology, vol. 301, pp. 188-198, 1998.

[58] A. Jordan and P. Reichard, "Ribonucleotide reductases," Annual Review of Biochemistry, vol. 67, pp. 71-98, 1998.

[59] A. Larsson and P. Reichard, "Allosteric effects and substrate specificity of the ribonucleoside diphosphate reductase system from Escherichia coli B," Biochimica et Biophysica Acta, vol. 113, no. 2, pp. 407-408, 1966.

[60] B. Roy, O. Guittet, C. Beuneu, G. Lemaire, and M. Lepoivre, "Depletion of deoxyribonucleoside triphosphate pools in tumor cells by nitric oxide," Free Radical Biology and Medicine, vol. 36, no. 4, pp. 507-516, 2004.

[61] D. Bogenhagen and D. A. Clayton, "Mouse 1 cell mitochondrial DNA molecules are selected randomly for replication throughout the cell cycle," Cell, vol. 11, no. 4, pp. 719-727, 1977.

[62] C. K. Mathews and S. Song, "Maintaining precursor pools for mitochondrial DNA replication," FASEB Journal, vol. 21, no. 10, pp. 2294-2303, 2007.

[63] G. Pontarin, L. Gallinaro, P. Ferraro, P. Reichard, and V. Bianchi, "Origins of mitochondrial thymidine triphosphate: dynamic relations to cytosolic pools," Proceedings of the National Academy of Sciences of the United States of America, vol. 100, no. 21, pp. 12159-12164, 2003.

[64] A. F. Davis and D. A. Clayton, "In situ localization of mitochondrial DNA replication in intact mammalian cells," Journal of Cell Biology, vol. 135, no. 4, pp. 883-893, 1996.

[65] S. Eriksson, B. Munch-Petersen, K. Johansson, and H. Ecklund, "Structure and function of cellular deoxyribonucleoside kinases," Cellular and Molecular Life Sciences, vol. 59, no. 8, pp. 1327-1346, 2002. 
[66] V. Bianchi and J. Spychala, "Mammalian5'-nucleotidases," The Journal of Biological Chemistry, vol. 278, no. 47, pp. 4619546198, 2003.

[67] A. Saada, A. Shaag, H. Mandel, Y. Nevo, S. Eriksson, and O. Elpeleg, "Mutant mitochondrial thymidine kinase in mitochondrial DNA depletion myopathy," Nature Genetics, vol. 29, no. 3, pp. 342-344, 2001.

[68] L. Wang, A. Saada, and S. Eriksson, "Kinetic properties of mutant human thymidine kinase 2 suggest a mechanism for mitochondrial DNA depletion myopathy," The Journal of Biological Chemistry, vol. 278, no. 9, pp. 6963-6968, 2003.

[69] C. Desler, B. Munch-Petersen, and L. J. Rasmussen, "The role of mitochondrial dNTP levels in cells with reduced TK2 activity," Nucleosides, Nucleotides and Nucleic Acids, vol. 25, no. 9\#8211;11, pp. 1171-1175, 2006.

[70] H. Tanaka, H. Arakawa, T. Yamaguchi et al., "A ribonucleotide reductase gene involved in a p53-dependent cell-cycle checkpoint for DNA damage," Nature, vol. 404, no. 6773, pp. 42-49, 2000.

[71] A. Bourdon, L. Minai, V. Serre et al., "Mutation of RRM2B, encoding p53-controlled ribonucleotide reductase (p53R2), causes severe mitochondrial DNA depletion," Nature Genetics, vol. 39, no. 6, pp. 776-780, 2007.

[72] R. L. Delsite, L. J. Rasmussen, A. K. Rasmussen, A. Kalen, P. C. Goswami, and K. K. Singh, "Mitochondrial impairment is accompanied by impaired oxidative DNA repair in the nucleus," Mutagenesis, vol. 18, no. 6, pp. 497-503, 2003.

[73] V. W. S. Liu, C. Zhang, and P. Nagley, "Mutations in mitochondrial DNA accumulate differentially in three different human tissues during ageing," Nucleic Acids Research, vol. 26, no. 5, pp. 1268-1275, 1998.

[74] K. K. Singh, M. Kulawiec, I. Still, M. M. Desouki, J. Geradts, and S.-I. Matsui, "Inter-genomic cross talk between mitochondria and the nucleus plays an important role in tumorigenesis," Gene, vol. 354, no. 1-2, pp. 140-146, 2005. 

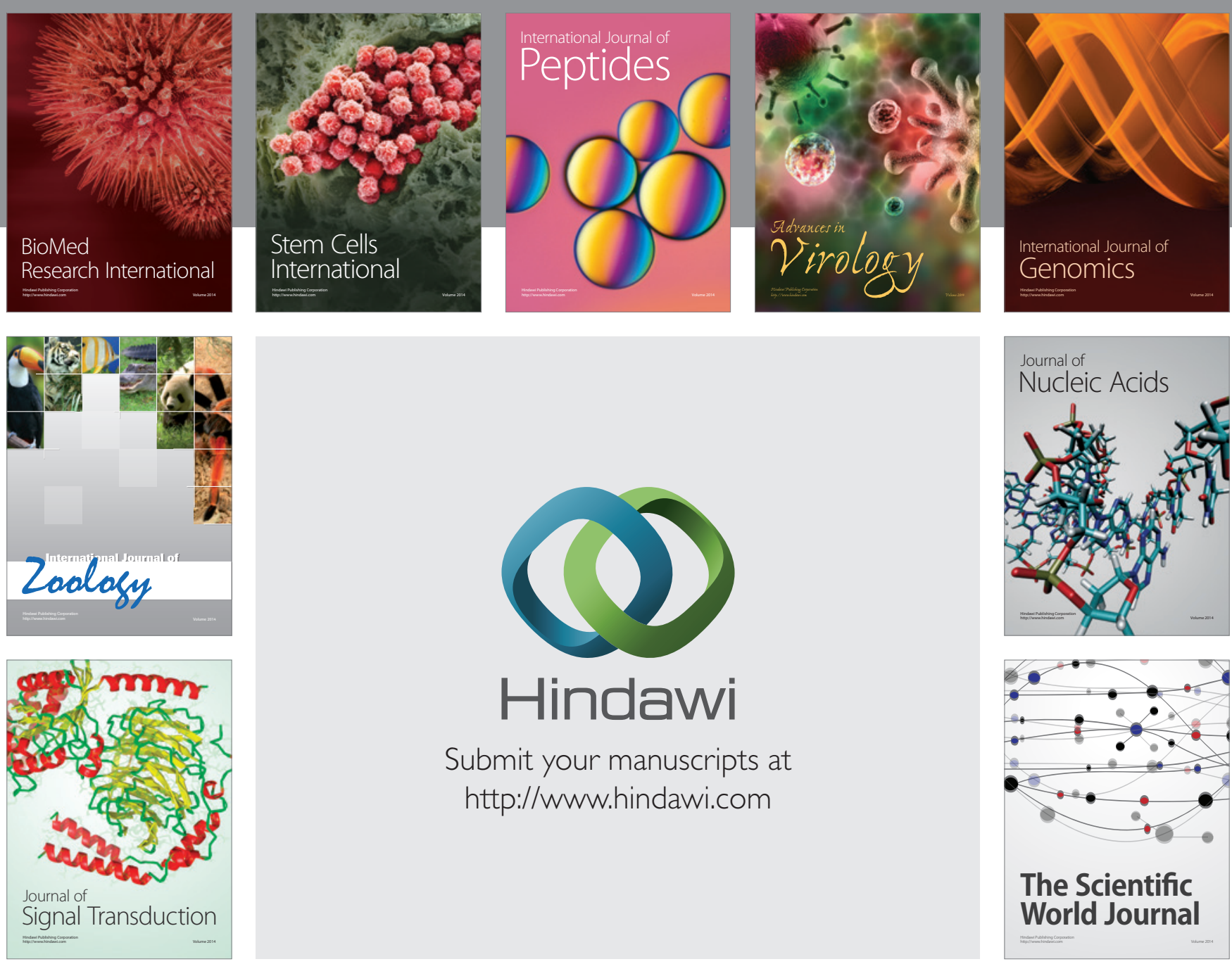

Submit your manuscripts at

http://www.hindawi.com
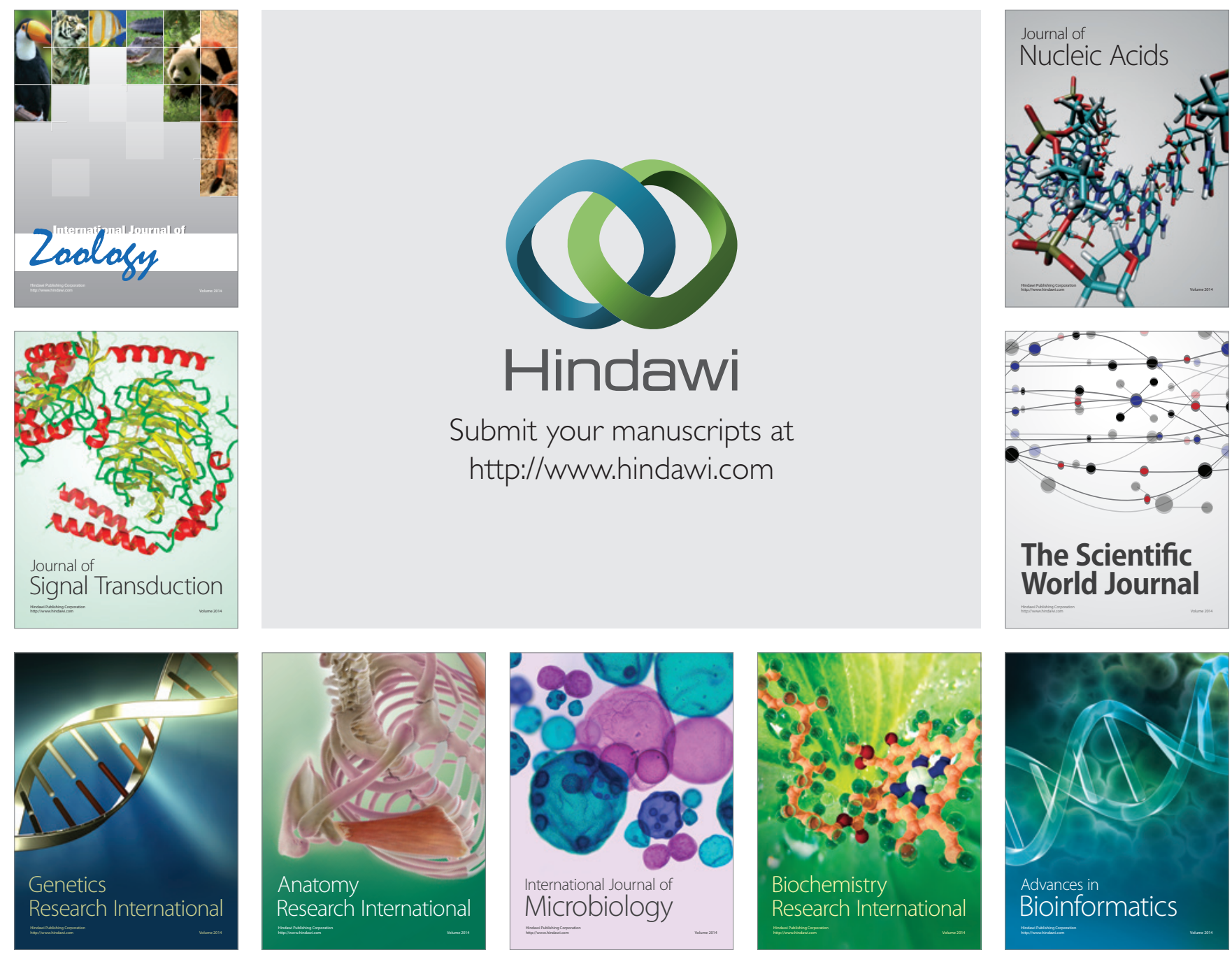

The Scientific World Journal
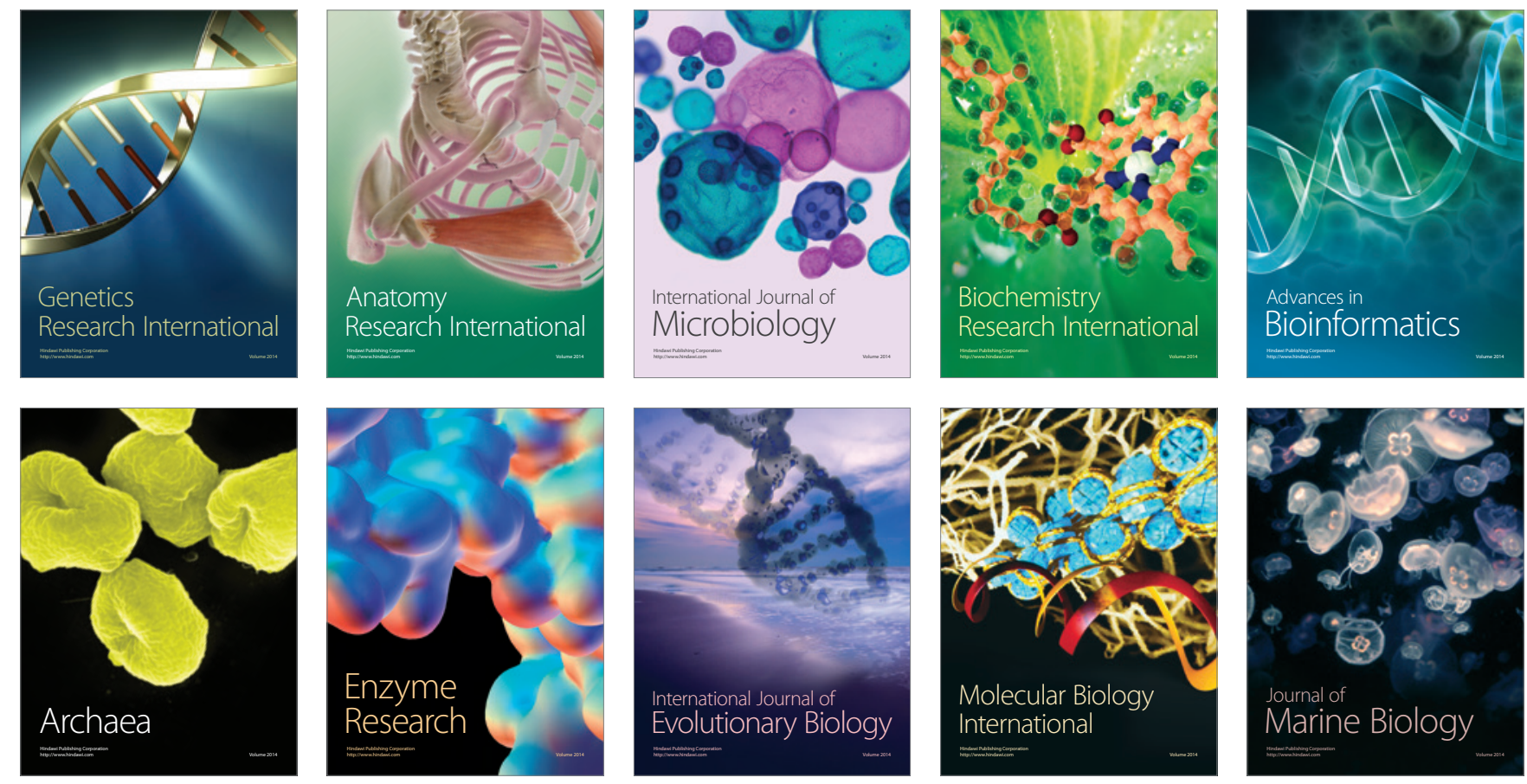